\title{
Effects of $\alpha$-lipoic acid on LPS-induced neuroinflammation and NLRP3 inflammasome activation through the regulation of BV-2 microglial cells activation
}

\author{
Su Min Kim ${ }^{1, \#}$, Ji Sun $\mathrm{Ha}^{1, \#}$, A Reum Han ${ }^{2}$, Sung-Woo Cho ${ }^{2, *}$ E Seung-Ju Yang ${ }^{1, *}$ \\ ${ }^{1}$ Department of Biomedical Laboratory Science, Konyang University, Daejeon 35365, ${ }^{2}$ Department of Biochemistry and Molecular Biology, \\ University of Ulsan College of Medicine, Seoul 05505, Korea
}

\begin{abstract}
Microglial cells are known as the main immune cells in the central nervous system, both regulating its immune response and maintaining its homeostasis. Furthermore, the antioxidant $\alpha$-lipoic acid (LA) is a recognized therapeutic drug for diabetes because it can easily invade the blood-brain barrier. This study investigated the effect of $\alpha$-LA on the inflammatory response in lipopolysaccharide (LPS)-treated BV-2 microglial cells. Our results revealed that $\alpha$-LA significantly attenuated several inflammatory responses in BV-2 microglial cells, including pro-inflammatory cytokines, such as tumor necrosis factor- $\alpha$ and interleukin (IL)-6, and other cytotoxic molecules, such as nitric oxide and reactive oxygen species. In addition, $\alpha$-LA inhibited the LPS-induced phosphorylation of ERK and p38 and its pharmacological properties were facilitated via the inhibition of the nuclear factor kappa B signaling pathway. Moreover, $\alpha$-LA suppressed the activation of NOD-like receptor pyrin domain containing 3 (NLRP3) inflammasomes, multiprotein complexes consisting of NLRP3 and caspase-1, which are involved in the innate immune response. Finally, $\alpha$-LA decreased the genes accountable for the M1 phenotype, IL-1 $\beta$ and ICAM1, whereas it increased the genes responsible for the M2 phenotype, MRC1 and ARG1. These findings suggest that $\alpha$-LA alleviates the neuroinflammatory response by regulating microglial polarization. [BMB Reports 2019; 52(10): 613-618]
\end{abstract}

*Corresponding authors. Seung-Ju Yang, Tel: +82-42-600-8432; Fax: +82-42-600-8408; E-mail: sjyang@konyang.ac.kr; Sung-Woo Cho, Tel: +82-2-3010-4278; Fax: +82-2-3010-4278; E-mail: swcho @amc.seoul.kr

${ }^{\#}$ These authors contributed equally to this work.

https://doi.org/10.5483/BMBRep.2019.52.10.026

Received 25 January 2019, Revised 7 February 2019, Accepted 12 February 2019

Keywords: $\alpha$-lipoic acid, Microglia, NLRP3 inflammasome, Polarization

\section{INTRODUCTION}

In pathological status, activation of microglial cells is essential in neuroinflammatory response as the resident phagocytes in the central nervous system (CNS) (1). Specifically, microglial cells are classified as either classically activated (M1 phenotype) or alternatively activated (M2 phenotype). Most previous studies indicated that microglial cells in the M1 phenotype release pro-inflammatory mediators, including cytokines, such as tumor necrosis factor (TNF)- $\alpha$ and interleukin (IL)-6, and other cytotoxic molecules, such as nitric oxide (NO) and reactive oxygen species (ROS) (2). In contrast, microglial cells in the M2 phenotype were shown to downregulate the release of pro-inflammatory cytokines and protect against inflammation (3). Therefore, the differentiation of microglial cells polarization suggests whether they are capable of inducing anti-inflammatory responses.

Furthermore, recent studies found both nuclear factor- $\mathrm{\kappa B}$ (NF-kB) and mitogen-activated protein kinase (MAPK) signaling to contribute to the activation of the nucleotide binding and oligomerization domain-like receptor containing a pyrin domain (NLRP3) inflammasome (4). NLRP3 inflammasomes are immune complexes consisting of NLRP3, the apoptosis-associated speck-like protein containing a C-terminal caspase recruitment domain (ASC), and pro-caspase-1. Although they regulate the immune response in microglial cells by activating both pro-caspase- 1 and interleukin (IL)- $1 \beta$ (5), the process by which NF-kB and MAPK signaling activate NLRP3 inflammasome is unknown.

The antioxidant $\alpha$-lipoic acid $(\alpha-\mathrm{LA})$ is considered an attractive drug candidate for anti-neuroinflammatory therapy. In fact, several studies reported the beneficial effects of $\alpha$-LA in various disorders, including hypertension (6), diabetes mellitus (7), while $\alpha$-LA was used as a safe supplement for humans in various countries. However, most studies on such $\alpha$-LA effects did not discuss microglial activation, specifically through NLRP3 inflammasome mediation.

In this study, we aimed to investigate the anti-inflammatory effects of $\alpha-L A$ in relation to its regulatory role on several inflammatory responses and NLRP3 inflammasome activation

ISSN: 1976-670X (electronic edition)

Copyright (C) 2019 by the The Korean Society for Biochemistry and Molecular Biology

(c) This is an open-access article distributed under the terms of the Creative Commons Attribution Non-Commercial License (http://creativecommons.org/licenses/by-nc/4.0) which permits unrestricted non-commercial use, distribution, and reproduction in any medium, provided the original work is properly cited. 
through increased M2 phenotype.

\section{RESULTS}

\section{$\alpha$-LA decreased pro-inflammatory cytokines in LPS-induced} BV-2 microglial cells

Cytotoxicity of $\alpha$-LA was evaluated prior to the analysis of pro-inflammatory cytokines in BV-2 cells. Cells were incubated with $\alpha-\mathrm{LA}(100,200,500$, and $1000 \mu \mathrm{M})$, with or without LPS $(1 \mu \mathrm{g} / \mathrm{ml})$, for $24 \mathrm{~h}$. $\alpha$-LA did not present cytotoxicity at any of the concentrations employed (Fig. 1A). These results suggest that $\alpha$-LA did not affect the viability of BV-2 cells in vitro. To further analyze the effects of $\alpha$-LA on pro-inflammatory cytokines production, BV-2 cells were incubated with both the indicated concentration of $\alpha$-LA and LPS. Although both TNF- $\alpha$ and IL- 6 levels were found to be increased in LPS-induced BV-2 cells, their expression was considerably decreased in $\alpha-$ LA treated BV-2 cells (Fig. 1B and C). These results suggest that $\alpha$-LA inhibits the production of pro-inflammatory cytokines without affecting cell viability.

Effects of $\alpha$-LA on both production of ROS and NO, and expression of NOS in LPS-stimulated BV-2 microglial cells The protective effect of $\alpha$-LA on LPS-induced ROS production was further investigated with H2DCF-DA. As shown in Fig. 2A, a $\sim 2$-fold increase in ROS production was found in LPS
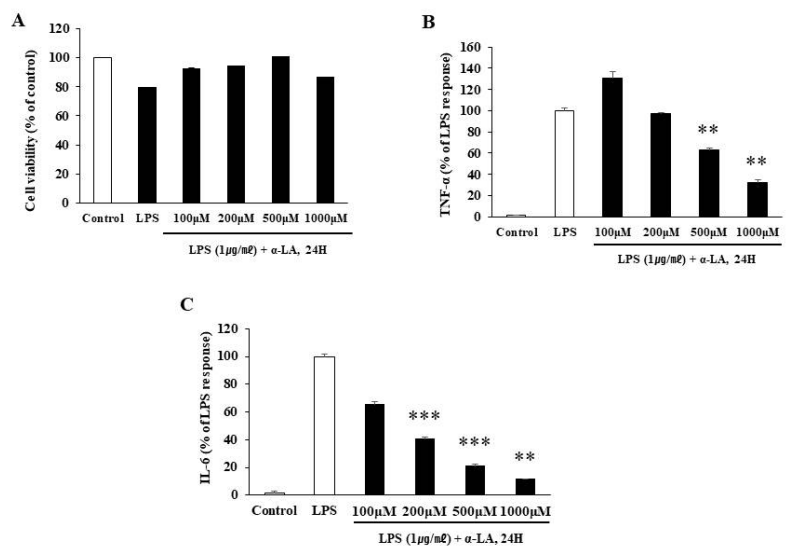

Fig. 1. $\alpha$-LA inhibits the expression of pro-inflammatory cytokines in LPS-treated BV-2 microglial cells. (A) Effects of $\alpha$-LA on cell viability. BV-2 microglial cells were incubated with LPS $(1 \mu \mathrm{g} / \mathrm{ml})$ for $30 \mathrm{~min}$ followed by treatment with the indicated concentrations of $\alpha$-LA for $24 \mathrm{~h}$. Thereafter, cell viability was assessed through the MTT assay. (B and C) BV-2 microglial cells were treated with LPS $(1 \mu \mathrm{g} / \mathrm{ml})$ for $30 \mathrm{~min}$ followed by treatment with the indicated concentrations of $\alpha$-LA at the suggested times. The cell-free conditioned culture medium was collected and analyzed with ELISA for TNF- $\alpha$, IL-6. Data from three independent experiments are presented as means \pm S.D. $* \leq 0.05, * *<0.01, * * *<0.001$ and are related to both LPS-induced cells and $\alpha$-LA treated cells. treated cells compared to its levels in untreated cells. However, the LPS-induced ROS production in BV-2 cells was significantly reduced by $\alpha$-LA (Fig. 2A). To test whether $\alpha$-LA has an anti-inflammatory effect on microglia, the level of NO production in BV-2 cell culture supernatant was measured. LPS was seen to highly increase the production of NO, whereas $\alpha$-LA treatment significantly blocked its formation (Fig. 2B). Successively, the effects of $\alpha$-LA on NOS expression were analyzed, considering its role as predominant pro-inflammatory enzyme and its correction in LPS-stimulated BV-2 cells. Furthermore, western blotting data indicated that $\alpha$-LA decreased the expression levels of NOS in LPS-treated BV-2 cells (Fig. 2C). Altogether, our results imply that $\alpha$-LA reduced the enhanced LPS-induced ROS generation and that it may inhibit NO formation, downregulating its expression.

$\alpha$-LA inhibits both phosphorylation of ERK and p38, and the activation of NLRP3 inflammasome and NF- $\mathrm{KB}$ in LPS-induced BV-2 cells

We examined whether the repressive effect of $\alpha$-LA is a result of the MAPK signaling pathways. Following the identification of an inhibition of the LPS-induced phosphorylation of both ERK and p38 by $\alpha$-LA (Fig. 3A), whether $\alpha$-LA could prevent the activation of the NF- $\mathrm{KB}$ pathway in LPS-treated BV-2 cells was explored. As shown in Fig. 3B, LPS stimulation provoked the translocation of NF- $\mathrm{KB}$ into the nucleus, whereas the $\alpha-\mathrm{LA}$ treatment blocked such a process. In addition, the LPS-induced phosphorylation of I $\mathrm{B} B \alpha$ was suppressed in $\alpha$-LA-treated BV-2 microglial cells (Fig. 3C).
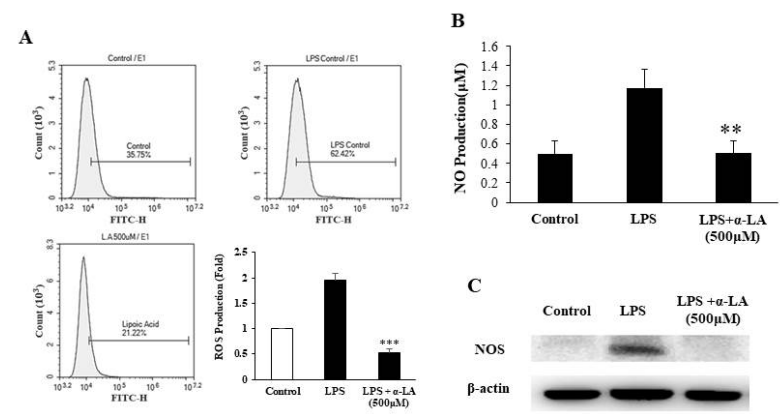

Fig. 2. Effects of $\alpha$-LA on both the production of ROS and NO, and the expression of NOS in LPS-stimulated BV-2 microglial cells. (A) LPS-induced ROS levels were measured through flow cytometry. $\mathrm{H}_{2}$ DCF-DA was treated during the last hour of incubation with both LPS $(1 \mu \mathrm{g} / \mathrm{ml})$ and $\alpha$-LA. (B) Cells were pretreated with LPS $(1 \mu \mathrm{g} / \mathrm{ml})$ followed by $\alpha$-LA for $24 \mathrm{~h}$, while the Griess reagent was used to measure NO. (C) NOS expression levels were detected by western blotting, while $\beta$-actin was used as the internal control. Densitometric analysis showed the suppression of NOS protein expression compared with LPS-induced BV-2 cells. Data from three independent experiments are presented as means \pm S.D. ${ }^{*} \mathrm{P}<0.05, * * \mathrm{P}<0.01$ and $* * * \mathrm{P}<$ 0.001 and are related to both LPS-induced cells and $\alpha$-LA treated cells. 

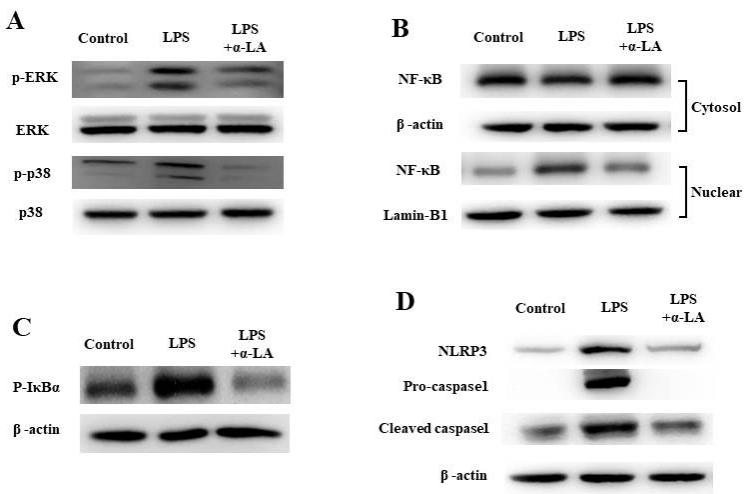

Fig. 3. Effects of $\alpha-L A$ on ERK, p38, NLRP3 inflammasome expression and NF- $\kappa B$ translocation in LPS-induced BV-2 microglial cells. BV-2 microglial cells were stimulated with LPS (1 $\mu \mathrm{g} / \mathrm{ml}$ ) for $30 \mathrm{~min}$ followed by treatment with the indicated concentrations of $\alpha$-LA for 2 hours. (A) BV-2 microglial cells were lysed to whole lysates. Activation of ERK and p38 was detected by western blotting. (B) The translocation of $N F-\kappa B$ was also analyzed through western blotting. BV-2 microglial cells were lysed to cytosolic extracts and nucleic extracts, while both $\beta$-actin and Lamin-B1 were used as internal controls. (C) LPS and $\alpha$-LA treatment was performed as described in the above legends. Finally, I $\mathrm{\kappa}-\mathrm{B} \alpha$ phosphorylation was detected by western blotting. (D) The western blot was performed to investigate the presence of NLRP3, pro-caspase-1 and cleaved caspase-1.

Previous studies have reported that the activation of NF- $\mathrm{\kappa B}$ mediates the activation of NLRP3 inflammasomes in microglia (4), which induces pro-caspase-1 cleavage. While western blot analysis demonstrated that NLRP3, pro-caspase-1, and active caspase-1 were markedly increased in response to LPS stimulation, treatment with $\alpha$-LA significantly reduced their LPS-induced protein expression when compared to those in control cells (Fig. 3D). This indicated that $\alpha$-LA, not only reduces the immune response associated with NF-kB signaling, but also decreased NLRP3 inflammasome activation.

\section{Effect of $\alpha$-LA on both the M1 and M2 phenotypes in LPS-induced BV-2 cells}

Microglia phenotype is distinguished by the expression of either the surface marker or specific molecules. Specifically, both ICAM-1 (CD54) and IL-1 $\beta$ are expressed in M1-positive microglial cells, whereas the MRC1 (CD206) surface marker and Th2-related molecules, such as Arginase 1, are expressed in M2 microglial cells (2). RT-qPCR and immunofluorescence analysis demonstrated that the transcription levels of both the ICAM-1 and IL-1 $\beta$ genes were upregulated in LPS-treated BV-2 microglial cells. However, $\alpha$-LA treatment effectively inhibited their expression levels (Fig. 4A and B, respectively). In contrast, $\alpha$-LA treatment slightly increased MRC1-positive cells in LPS-induced BV-2 cells (Fig. 4C). Similarly, the expression of ARG1 mRNA was increased in all $\alpha$-LA treated cells when compared to LPS-induced BV-2 cells (Fig. 4D). These results
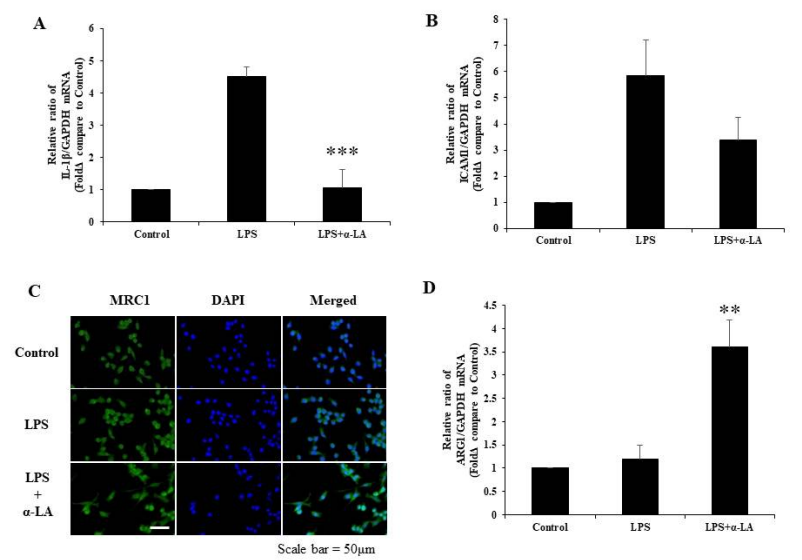

Fig. 4. $\alpha$-LA inhibits the expression of pro-inflammatory cytokines in M1 polarized BV-2 microglial cells following LPS treatment. The effects of $\alpha-L A$ on microglial polarization was analyzed through both immunofluorescence and RT-qPCR. BV-2 microglial cells were treated with LPS $(1 \mu \mathrm{g} / \mathrm{ml})$ for $30 \mathrm{~min}$ and by indicated concentrations of $\alpha-\mathrm{LA}$ at the suggested times. (A, B and D) The cytokine IL-1 $\beta$, the cell surface marker ICAM1 and ARG1 mRNA expression in the total RNA were assessed through real-time quantitative PCR. (C) BV-2 microglial cells were visualized using an anti-MRC1 antibody and an Alexa Fluor ${ }^{(R)}$ 488-linked secondary antibody. Successively, coverslips were detected via fluorescence microscopy. Scale bar $=100 \mu \mathrm{m}$.

indicate that $\alpha$-LA switched microglia cells phenotype from $\mathrm{M} 1$ to $\mathrm{M} 2$.

\section{DISCUSSION}

Several studies have indicated that $\alpha$-LA exerts a neuroprotective effect in several neurologic diseases, Alzheimer's disease (8). Moreover, LPS-induced microglial cells damage neurons by releasing ROS, NO, and other cytokines such as TNF- $\alpha$ and IL-6 (9). ROS, a major factor in the pathological features of neurodegenerative diseases, may be linked to activation of NLRP3 inflammasome and IL-1 $\beta$ expression $(10,11)$. Also, elevated $\mathrm{NO}$ levels contributes to nitrative stress and NOS is the key enzyme responsible for NO production after exposure to LPS (12). Our results confirmed the effect of $\alpha$-LA on microglial cell activation following LPS treatment, considering that the $\alpha$-LA treated group reported significantly decreased ROS, NO, and NOS expression and inhibition of TNF- $\alpha$ and IL-6.

Further, NLRP3 inflammasome are highly organized intracellular sensors for neuronal alarming signals, such as DAMP, LPS, and $\beta$-amyloid. They consist of NLRP3, ASC, and pro-caspase-1, which activate both caspase-1 and IL-1 $\beta$ when stimulated (13). Additionally, our group showed in previous studies that NLRP3 inflammasome expressed in LPS-stimulated BV-2 microglial cells can be reduced by drugs (14). Surprisingly, the present study confirmed the effect of $\alpha$-LA on 
NLRP3 inflammasome activation. Specifically, our data indicated that $\alpha$-LA considerably reduced NLRP3, pro-caspase- 1 and cleaved caspase-1. Considering that various studies suggested that NLRP3 inflammasome activate NF- $\kappa$ B, MAPK signaling, and ROS, renowned inflammatory responses $(15,16)$, the effect of $\alpha$-LA on the regulation of the NF-kB and MAPK signaling pathway was investigated. Other studies showed that LPS stimulates the phosphorylation of p38 and JNK not ERK, or only p38 $(17,18)$. In this study, LPS activated p38 and ERK MAPKs in BV-2 microglial cells as observed previously (18). Simultaneously, our findings reported the inhibition of both NF- $\mathrm{KB}$ translocation to the nucleus and MAPK signaling molecules, such as p-ERK and p-p38, in $\alpha$-LA-treated BV-2 microglial cells.

Activated microglial cells present the classical activation (M1 phenotype), which is associated with pro-inflammatory mediators, including IL-1 $\beta$, IL-6, and TNF- $\alpha$. In contrast, those with the alternative activation (M2 phenotype) are associated with the induction of specific proteins, such as Arg1 and the MRC1 (19). Considering that microglial activation is important in several chronic neurodegenerative diseases, such as Alzheimer's disease and Parkinson's disease $(20,21)$, monitoring microglial activation may represent an innovative proposal for the treatment of neurodegenerative disease. Moreover, the NLRP3 inflammasome-related components links with the changes in the polarization state of macrophage (22). In fact, our results suggest $\alpha$-LA-treated LPS-stimulated BV-2 microglial cells have reduced markers of the M1 phenotype, namely the IL-1 $\beta$ and ICAM1, whereas the M2 phenotype markers, i.e., MRC1 and ARG1, were higher in the group treated with $\alpha$-LA than in that stimulated with LPS. $\alpha$-LA is involved in controlling M1 polarization through IL-1 $\beta$, a key proinflammatory cytokine produced by inflammasome activation in BV-2 microglial cells. Furthermore, increased expression level of ARG1 mRNA and MRC1 protein shows that $\alpha$-LA inhibits NLRP3 inflammasome activation and promote M1 microglia induced by LPS toward M2 phenotype.

In summary, the current study suggests that $\alpha$-LA both reduces NLRP3 inflammasome activation, NF- $\mathrm{B}$, MAPK signaling, and pro-inflammatory cytokine release in LPSinduced BV-2 microglial cells and modulates microglial cells M1/M2 polarization. Although the mechanism underlying the regulation of NLRP3 inflammasome activation by microglial cells polarization is yet to be fully elucidated, our study indicates that $\alpha$-LA may represent a candidate drug for the treatment of both inflammatory and chronic neurodegenerative diseases.

\section{MATERIALS AND METHODS}

\section{Materials}

Both $\alpha$-lipoic acid and lipopolysaccharide (LPS) were obtained from Sigma-Aldrich (St. Louis, MO, USA). In contrast, Dulbecco's modified Eagle's medium (DMEM) and phosphate- buffered saline (PBS) were purchased from Hyclone (Logan, UT, USA). Penicillin $(100 \mathrm{U} / \mathrm{ml}) /$ streptomycin $(100 \mu \mathrm{g} / \mathrm{ml})$ was purchased from Gibco (Life Technologies Inc., Gaithersburg, $M D$, USA), and heat-inactivated fetal bovine serum (FBS) was obtained from Pan Biotech (Aidenbach, Germany). Both TNF- $\alpha$ and IL-6 were quantitatively measured through an enzyme-linked immunosorbent assay (ELISA) using the mouse TNF- $\alpha$ and IL- 6 DuoSet ELISA kit (R\&D systems, Minneapolis, $M N$, USA), according to the manufacturer's instructions. Additionally, intracellular ROS production was estimated with $\mathrm{H}_{2}$ DCF-DA from molecular probes (Carlsbad, OR, USA). The following antibodies were used for western blotting: anti-NOS, anti-ERK, anti-pERK, anti-p38, anti-p-p38, anti-NF-кB, anti-IкB $\alpha$, anti-p-l $\kappa B \alpha$, anti-NLRP3 (Cell Signaling Technology, Beverly, MA, USA), anti-caspase-1 (p20), anti-ASC (AdipoGen life science, USA) anti-JNK, anti-p-JNK, anti-caspase- 1 , anti- $\beta$-actin and anti-Lamin B1 (Santacruz Biotechnology, Santa Cruz, CA, USA). In contrast, the antibodies employed for immunofluorescence labeling analysis are the anti-Mannose receptor, goat anti-mouse IgG H\&L Alexa Fluor ${ }^{R} 488$, and goat anti-rabbit IgG H\&L Alexa Fluor ${ }^{\mathbb{R}} 488$ (Abcam, Milton, Cambridge, UK). All cells were counterstained with DAPI (Thermo Fisher Scientific, Pittsburgh, PA, USA).

\section{Cell culture}

The BV-2 microglial cell line was obtained from the Department of Biochemistry and Molecular Biology, University of Ulsan College of Medicine (Seoul, South Korea) and was maintained in DMEM with penicillin $(100 \mathrm{U} / \mathrm{ml})$, streptomycin $(100 \mu \mathrm{g} / \mathrm{ml})$, and $10 \% \mathrm{FBS}$ at $37^{\circ} \mathrm{C}$. BV-2 cells were incubated in a humidified atmosphere containing $5 \% \mathrm{CO}_{2}$.

\section{MTT assay}

BV-2 microglial cells were seeded in a 96-well plate $\left(1 \times 10^{4}\right.$ cells/well) and cultured overnight. Successively, they were incubated with LPS $(1 \mu \mathrm{g} / \mathrm{ml})$ for $30 \mathrm{~min}$, prior to the addition of the $\alpha$-lipoic acid $(100,200,500$, and $1000 \mu \mathrm{M})$. After incubation for $24 \mathrm{~h}$, cells were assayed for cell viability by adding the Ez-Cytox reagent $(20 \mu \mathrm{l} /$ well) and further incubated for $1 \mathrm{~h}$ at $37^{\circ} \mathrm{C}$. Thereafter, the 96 -well plate was measured with an enzyme-linked immunosorbent assay microplate reader (Molecular Device, Sunnyvale, CA, USA) at $450 \mathrm{~nm}$.

\section{Enzyme-linked immunosorbent assay}

BV-2 microglial cells were seeded in a 6 -well plate $\left(5 \times 10^{5}\right.$ cells/well) and incubated with LPS $(1 \mu \mathrm{g} / \mathrm{ml})$ for $30 \mathrm{~min}$, followed by treatment with $\alpha$-LA $(500 \mu \mathrm{M})$. After incubation for $24 \mathrm{~h}$, cell-free supernatants were collected and both TNF- $\alpha$ and IL- 6 levels were measured in the extracellular medium with the mouse TNF- $\alpha$ and IL- 6 DuoSet ELISA kit, according to the manufacturer's instructions. Absorbance was measured at $450 \mathrm{~nm}$ using the enzyme-linked immunosorbent assay microplate reader (23). All assays were performed in three independent experiments. The concentration of both TNF- $\alpha$ 
and IL-6 was calculated employing the standard value obtained from a linear regression equation.

\begin{abstract}
Measurement of ROS through flow cytometry
The measurement of DCF was performed to estimate the generation of intracellular ROS in BV-2 microglial cells. Briefly, BV-2 microglial cells were seeded in a 6-well plate (5 $\times 10^{5}$ cells/well) and cultured overnight. Thereafter, they were incubated with LPS $(1 \mu \mathrm{g} / \mathrm{ml})$ for 30 min prior to the treatment of $\alpha$-LA $(500 \mu \mathrm{M})$. After incubation for 24 h, H2DCF-DA was treated during the last hour of incubation. Successively, cells were harvested and washed twice with PBS. Finally, ROS levels were analyzed through flow cytometry (NOVOCYTE flow cytometer, ACEA Biosciences Inc). Data were analyzed using the Novoexpress software (ACEA Biosciences Inc).
\end{abstract}

\section{Measurement of nitric oxide through a biochemical assay}

The NO concentration in the conditioned culture medium was indirectly assessed by measuring the amount of generated nitrite. BV-2 microglial cells were seeded in a 6 -well plate (5 $\times 10^{5}$ cells/well) and incubated with LPS $(1 \mu \mathrm{g} / \mathrm{ml})$ for $30 \mathrm{~min}$, followed by treatment with $\alpha$-LA $(500 \mu \mathrm{M})$ for $24 \mathrm{~h}$. Cell-free conditioned culture medium was collected and measured using the nitric oxide detection kit, based on the Griess method.

\section{Western blot analysis}

Following the treatment with both LPS $(1 \mu \mathrm{g} / \mathrm{ml})$ and $\alpha$-LA $(500 \mu \mathrm{M})$ at their specific times, BV-2 microglial cells pellets were lysed using either the cytosolic extraction or RIPA buffer. Thereafter, protein samples (30 $\mu \mathrm{g} / \mathrm{lane})$ were separated by $10-12 \%$ SDS-polyacrylamide gel. After transfection at the nitrocellulose membrane, they were incubated overnight at $4^{\circ} \mathrm{C}$ with the primary antibody, which was followed by incubation for $1 \mathrm{~h}$ at room temperature with either the anti-rabbit IgG HRP or the anti-mouse IgG HRP secondary antibodies. The membrane was developed using the enhanced chemiluminescence detection system (Vilber Lourmat, Marne la Vallee, France).

\section{Immunofluorescence analysis}

BV-2 microglial cells were grown on coverslips and treated with LPS $(1 \mu \mathrm{g} / \mathrm{ml})$ for $30 \mathrm{~min}$ followed by treatment with $\alpha$-LA at the indicated times. Thereafter, cells were fixed with $4 \%$ paraformaldehyde at room temperature for $10 \mathrm{~min}$ and permeabilized with $0.25 \%$ Triton $\mathrm{X}-100$ at room temperature for additional $10 \mathrm{~min}$. Successively, they were blocked with $1 \%$ BSA, $22.52 \mathrm{mg} / \mathrm{ml}$ glycine in $0.1 \%$ PBST solution for 30 min at room temperature. Subsequently, the coverslips containing cells were incubated with the anti-MRC1 (Abcam, Milton, Cambridge, UK) antibody at $4^{\circ} \mathrm{C}$ overnight and the corresponding secondary antibody at room temperature for an additional hour. Finally, nuclei were counterstained with DAPI and cells were observed through a fluorescence microscope
(Eclipse Ts2R, Nikon, Tokyo, Japan).

\section{Real-Time quantitative PCR analysis}

BV-2 microglial cells were harvested and lysed in the TRIzol (Life Technologies) reagent, according to the manufacturer's instructions, and quantified using the NanoDrop ${ }^{\mathrm{TM}}$ One. The synthesis of the cDNA template was performed with the DiaStar $^{\text {TM }}$ 2X RT Pre-Mix (SolGent). Quantitative RT-PCR (qRT-PCR) was accomplished using GAPDH as an internal control to normalize the expression level. Real-time PCR was conducted in a CFX96 ${ }^{\mathrm{TM}}$ real-time system employing the SsoAdvanced ${ }^{\mathrm{TM}}$ Universal SYBR ${ }^{\mathbb{R}}$ Green Supermix for CDNA quantification. Furthermore, the following primers were used: IL-1 $\beta$ (Forward- GCCCATCCTCTGTGACTCAT, Reverse- AGG CCACAGGTATTTTGTCG), ICAM-1 (Forward- AGCACCTCCC CACCTACTIT, Reverse- AGCTTGCACGACCCTTCT AA), ARG1 (Forward- GTGAAGAACCCACGGTCTGT, Reverse- CTGGT TGTCA GGGGAGTGTT) and GAPDH (Forward- TGTCCGTC GTGGATCTGAC, Reverse- CCTGCTTCACCACCTTCTTG).

\section{Statistical analyses}

Data were presented as means \pm standard error and were representative of three independent experiments. SPSS statistical software package (Version 18.0, Chicago, IL, USA) was used for analysis of variance (ANOVA), as appropriate. Additionally, individual differences among each group were compared through one-way ANOVAs, followed by the Scheffe method and Dunnett T3 method. A threshold of $\mathrm{P}<0.05$ was considered statistically significant.

\section{ACKNOWLEDGEMENTS}

This work was supported by the Basic Science Research Program through the National Research Foundation of Korea (NRF) funded by the Ministry of Education (2018R1D1A 3A03000692) and by the National Research Foundation of Korea (NRF) grant funded by the Korea government (MSIT) (2018R1A2B6001743).

\section{CONFLICTS OF INTEREST}

The authors have no conflicting interests.

\section{REFERENCES}

1. Shi H, Sheng B, Zhang F et al (2013) Kruppel-like factor 2 protects against ischemic stroke by regulating endothelial blood brain barrier function. Am J Physiol Heart Circ Physiol 304, H796-805

2. Cunha C, Gomes C, Vaz AR and Brites D (2016) Exploring new inflammatory biomarkers and pathways during LPS-Induced M1 polarization. Mediators Inflamm 2016, 6986175

3. Orihuela R, McPherson CA and Harry GJ (2016) Microglial M1/M2 polarization and metabolic states. $\mathrm{Br}$ J 
Pharmacol 173, 649-665

4. Bauernfeind FG, Horvath G, Stutz A et al (2010) NF-kB activating pattern recognition and cytokine receptors license NLRP3 inflammasome activation by regulating NLRP3 expression. J Immunol 183, 787-791

5. Jo EK, Kim JK, Shin DM and Sasakawa C (2016) Molecular mechanisms regulating NLRP3 inflammasome activation. Cell Mol Immunol 13, 148-159

6. Thirunavukkarasu V, Anitha Nandhini AT and Anuradha CV (2004) Lipoic acid attenuates hypertension and improves insulin sensitivity, kallikrein activity and nitrite levels in high fructose-fed rats. J Comp Physiol B 174, 587-592

7. Kamenova P (2006) Improvement of insulin sensitivity in patients with type 2 diabetes mellitus after oral administration of alpha-lipoic acid. Hormones (Athens) 5, 251-258

8. Maczurek AE, Ooi L, Patel M and Münch G (2013) Lipoic acid as an anti-inflammatory and neuroprotective treatment for Alzheimer's disease. Adv Nat Med Nutraceuticals Neurocognition 60, 71-88

9. Brown GC and Vilalta A (2015) How microglia kill neurons. Brain Res 1628, 288-297

10. Qureshi GA, Baig S, Sarwar M and Parvez SH (2004) Neurotoxicity, oxidative stress and cerebrovascular disorders. Neurotoxicology 25, 121-138

11. Chen L, Na R, Boldt E and Ran Q (2015) NLRP3 inflammasome activation by mitochondrial reactive oxygen species plays a key role in long-term cognitive impairment induced by paraquat exposure. Neurobiol Aging 36, 2533-2543

12. Chew LJ, Takanohashi A and Bell M (2006) Microglia and inflammation: impact on developmental brain injuries. Ment Retard Dev Disabil Res Rev 12, 105-112

13. Sorbara MT and Girardin SE (2011) Mitochondrial ROS fuel the inflammasome. Cell Res 21, 558-560
14. Yang JW, Yang SJ, Na JM, Hahn HG and Cho SW (2018) 3-(Naphthalen-2-yl(propoxy)methyl)azetidine hydrochloride attenuates NLRP3 inflammasome-mediated signaling pathway in lipopolysaccharide-stimulated BV2 microglial cells. Biochem Biophys Res Commun 495, 151-156

15. Walsh JG, Muruve DA and Power C (2014) Inflammasome in the CNS. Nat Rev Neuroeci 15, 84-97

16. Fan $\mathrm{HH}$, Zhu LB, Li T et al (2017) Hyperoside inhibits lipopolysaccharide-induced inflammatory responses in microglial cells via p38 and NFKB pathways. Int Immunopharmacol 50, 14-21

17. Yang XW, Li YH, Zhang H et al (2016) Safflower Yellow regulates microglial polarization and inhibits inflammatory response in LPS-stimulated Bv2 cells. Int J Immunopathol Pharmacol 29, 54-64

18. Kim EA, Han AR, Choi J, Ahn JY, Choi SY and Cho SW (2014) Anti-inflammatory mechanisms of $\mathrm{N}$-adamantyl-4methylthiazol-2-amine in lipopolysaccharide-stimulated BV-2 microglial cells. Int Immunopharmacol 22, 73-83

19. Heneka MT, Carson MJ, El Khoury J et al (2015) Neuroinflammation in Alzheimer's disease. Lancet Neurol 14, 388-405

20. Tremblay MÈ and Sierra A (2014) Microglia in health and disease. Microglia Heal Dis 313, 1-486

21. Kim SH, Oh K, Jin HK and Bae J (2018) Immune inflammatory modulation as a potential therapeutic strategy of stem cell therapy for ALS and neurodegenerative diseases. BMB Rep 51, 545-546

22. Samstad EO, Niyonzima N, Nymo S et al (2014) Cholesterol crystals induce complement-dependent inflammasome activation and cytokine release. J Immunol 192, 2837-2845

23. Yang SJ, Kim J, Lee S et al (2017) Anti-inflammatory and anti-oxidative effects of on beta-amyloid-induced microglial activation. BMB Rep 50, 634-639 